\title{
Introducción histórica a la física del caos
}

DOI: $10.31978 / 014-18-009-X .02$

\author{
CARlos Santos Burguete \\ Centro Nacional de Predicción (CNP), Agencia Estatal de Meteorología (AEMET)
}

DAVID FUERTES MARRÓN

Instituto de Energía Solar, Escuela Técnica Superior de Ingenieros de Telecomunicaciones (ETSIT), Universidad

Politécnica de Madrid (UPM)

Si conociéramos exactamente las leyes de la naturaleza y la situación del universo en el momento inicial, podríamos predecir exactamente la situación de ese mismo universo en un instante posterior. Pero incluso si las leyes naturales no tuvieran secretos para nosotros, sólo podríamos conocer la situación inicial aproximadamente. Si eso nos permitiera predecir la situación posterior con la misma precisión, es todo lo que necesitamos y deberíamos decir que el fenómeno se ha previsto, que está gobernado por leyes. Pero no es siempre así: puede suceder que pequeñas diferencias en las condiciones iniciales produzcan grandes diferencias en el fenómeno posterior. Un pequeño error al principio produciría un enorme error al final. La predicción se vuelve imposible y estamos ante el fenómeno fortuito.

Ciencia y Método, 1903 - Henri PoInCARÉ

¿Cómo se fraguó la física del caos? En este capítulo introducimos esta teoría, encuadrándola dentro de los grandes paradigmas en la historia de la ciencia.

Palabras clave: historia de la física del caos, física del caos, paradigmas científicos, determinismo, indeterminismo.

Imagen parte superior: recreación artística de JUAN CARLOS GARCíA COLOMBO. 


\subsection{Paradigmas científicos}

El método científico es una poderosa aproximación para ayudarnos a entender el mundo que nos rodea, dando las teorías científicas una explicación a algunos aspectos de la realidad. Para ello las teorías deben ser capaces de predecir el comportamiento de los citados aspectos la naturaleza y, para poder ser aceptadas, deben ser demostradas experimentalmente o, al menos, confirmadas mediante hechos u observaciones.

Tal y como fue descrito por FRANCIS BACON en 1620 en la obra Novum organum (Indicaciones relativas a la interpretación de la naturaleza [1]), el método científico puede entenderse como una receta que resume el procedimiento, basado en la lógica y la razón (cualidades humanas y no divinas), mediante el cual el hombre sería capaz de comprender y dominar (hoy en día debería cambiarse este dominar por armonizar con) la naturaleza. La receta se basa, de manera resumida, en la secuencia ordenada de los siguientes pasos: la observación de los fenómenos naturales; la elaboración de hipótesis capaces de explicar lo observado; y la experimentación, que deberá demostrar o refutar dichas hipótesis. El resultado final es una teoría científica que deberá ser capaz, por una parte, de explicar acontecimientos pasados (los fenómenos naturales observados) y, por otra, de predecir acontecimientos futuros (fenómenos por ocurrir).

Como se puede deducir de lo anterior, la importancia del procedimiento es mayúscula. Por una parte, el método científico nos permite proyectar hacia el futuro lo que vamos aprendiendo del pasado y del presente: es aquí donde la palabra predicción, habitual en este libro, cobra todo su sentido. Por otra, el método es un sistema capaz de autocorregirse, al incorporar el procedimiento para sustituir teorías científicas que se demuestran incapaces de seguir explicando la realidad observada por otras versiones mejoradas. Por último, y no por ello menos importante, el método científico proporciona el mejor antídoto frente a dogmas y prejuicios de toda clase, ya que el uso de la lógica y de la razón fundamenta el pensamiento crítico. A partir de la propuesta de BACON el mundo occidental comenzó a cuestionarse las cosas y dejó simplemente de aceptar las verdades aristotélicas asumidas hasta entonces, verdades que habían imposibilitado en buena medida la mejora de las condiciones de vida de gran parte de la humanidad.

En el sentido descrito por KARL R. POPPER, fundador del falsacionismo, una teoría, para ser científica debe poder ser falsable, es decir, susceptible de probarse su veracidad o falsedad.

Falsacionismo y teoría de la demarcación. Para constatar una teoría se debe intentar refutarla mediante un contraejemplo [17]. Pero no todas las teorías pueden demostrarse con la misma rotundidad, no tienen un apoyo experimental igual, ni son falsables de una forma igual de clara, ni tienen el mismo soporte matemático. Por ejemplo, la teoría electromagnética de JAMES C. MAXWELL es apta para reproducir experimentos y demostrarse experimentalmente en un laboratorio. Para la relatividad general de ALBERT EINSTEIN pueden diseñarse experimentos, pero serán más complicados y costosos. La selección natural de CHARLES DARWIN no permite reproducir experimentos en un laboratorio del mismo modo aunque hay numerosas observaciones consistentes con la teoría que apoyan su veracidad. De un modo similar, la cosmología se basa casi exclusivamente en observaciones pasivas: no podemos reproducir el universo entero en un laboratorio pero podemos examinar con ojo crítico la consistencia de la teoría con las observaciones. Importantes filósofos de la ciencia han explorado el terreno de la falsabilidad y el llamado Problema de la demarcación, dibujar una frontera entre el conocimiento científico y el no científico. LAKATOS [9] con sus programas de investigación y FEYERABEND [6] con su teoría anarquista de la ciencia matizan y enriquecen el falsacionismo de POPPER. MARIO BUNGE, filósofo de la ciencia argentino, establece una visión crítica sobre el concepto de falsabilidad de Popper [2]. Edgar Morin también, con un enfoque multidisciplinar y multirreferenciado [15]. En este terreno no hay un consenso universal y muchos científicos mantienen un punto de vista pragmático: las teorías pueden ser falsables y deben ser confirmables. Podemos ir más allá y cuestionar el concepto mismo de ley física [4]. En la actualidad hay enfoques muy refinados, e. g. DEBORAH MAYO construye un nuevo experimentalismo, contribuyendo a tratar tanto el relativismo como el llamado problema de Duhem, relacionado con los casos en los que una predicción científica no es consistente con el experimento [12, 13]. 
Existe un amplio y rico mosaico de teorías científicas en el que podemos encontrar teorías más o menos experimentales, teorías más o menos falsables, teo- rías con mayor o menor soporte matemático, etc. La Tabla 2.1 ofrece una relación cronológica de algunas personalidades en filosofía de la ciencia.

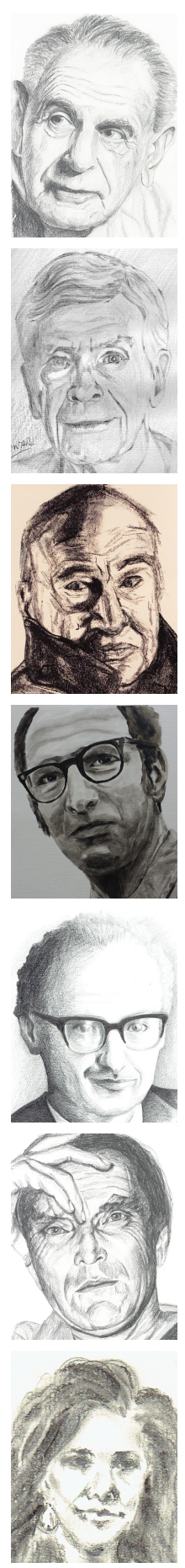

KARL RAIMUND POPPER (1902-1994), filósofo austriaco-británico, rechazando el inductivismo clásico del método científico postuló el falsacionismo empírico. Una teoría científica debe ser falsable, es decir, susceptible de demostrarse su falsedad.

Mario Augusto Bunge (1919-), físico, filósofo, epistemólogo y humanista argentino; realista, cientificista, materialista y sistemista; crítico con pseudociencias (psicoanálisis, praxeología, la homeopatía, la microeconomía neoclásica), existencialismo, fenomenología, posmodernismo, hermenéutica y feminismo filosófico.

EDGAR MORIN (1922-), filósofo y sociólogo francés, defiende el enfoque multidisciplinar y multirreferenciado para abordar la complejidad, «la innovación requiere un relajamiento de tensiones con un principio reorganizado».

ThOMAS SAMUEL KuHN (1922-1996), físico, historiador y filósofo de la ciencia estadounidense, contribuyó al cambio de orientación de la filosofía y sociología científicas. Describió las revoluciones científicas como cambios de paradigma provocados por las crisis.

IMRE LAKATOS (1922-1974), matemático y filósofo de la ciencia húngaro, recoge y critica a POPPER y a KUHN. Mejora lo que él llama falsacionismo dogmático y falsacionismo ingenuo, con el falsacionismo sofisticado, dando importancia a la confirmación de las teorías.

PAUl KARL FEYERABEND (1924-1994), filósofo de la ciencia austriaco, popperiano, antirracionalista, sucesivamente empirista y antiempirista, antipositivista y relativista, postuló el anarquismo epistemológico, co-autor de la tesis de la inconmensurabilidad, famoso por no utilizar un lenguaje formal.

DEBORAH G. MAYO (1951-), filósofa y socióloga de la ciencia estadounidense, ha contribuído en subdeterminación, el papel de nuevas evidencias, el problema de Duhem, la naturaleza del progreso científico y el análisis de riesgo.

Tabla 2.1: Algunos filósofos de la ciencia. 


\subsection{El determinismo}

Antes de la aparición de la teoría cuántica o la teoría del caos, el determinismo fue el paradigma imperante en física desde Newton y Laplace. PierreSIMON LAPLACE, astrónomo, físico y matemático francés (conocido como el NEWTON de Francia), a pesar de haber sentado las bases de la teoría de la probabilidad [10] fue un ferviente partidario del determinismo, como revela la siguiente cita:

«Podemos mirar el estado presente del universo como el efecto del pasado y la causa de su futuro. Se podría concebir un intelecto que en cualquier momento dado conociera todas las fuerzas que animan la naturaleza y las posiciones de los seres que la componen; si este intelecto fuera lo suficientemente vasto como para someter los datos a análisis, podría condensar en una simple fórmula el movimiento de los grandes cuerpos del universo y del átomo más ligero; para tal intelecto nada podría ser incierto y el futuro, así como el pasado, estarían frente a sus ojos» [10] (tal intelecto sería el famoso Demonio de Laplace).

Posteriormente, y dentro de la propia física clásica, surgieron otras objeciones bien fundamentadas al determinismo: según el ingeniero químico ROBERT UlANOWICZ, la termodinámica es el ejemplo más importante, acuñando conceptos como irreversibilidad y entropía, o la segunda ley de la termodinámica, relacionada con la flecha del tiempo [20].

Todavía en el marco del determinismo, se ha propuesto la existencia de un límite en el poder computacional del universo, es decir en la capacidad del demonio de LAPLACE para almacenar una cantidad infinita de información. Este límite estaría relacionado con la entropía máxima del universo, la velocidad de la luz y el tiempo mínimo necesario para mover información a través de la longitud de Planck. El valor de dicho límite rondaría los $10^{120}$ bits $[11,14]$. Por tanto, la evolución de un sistema con más datos no podría ser computada aunque se dispusiera de toda la edad del universo para hacerlo. Existe, al parecer, una limitación básica en el universo relacionada con esta idea, al igual que existe la limitación que impone el principio de incertidumbre de HEISENBERG en física cuántica: no pueden medirse simultáneamente la posición y el momento (por ende, la velocidad) de una partícula elemental con una precisión absoluta. Estas últimas ideas enlazan con algunas filosofías orientales: una parte del tao no puede explicar el tao y por tanto, en ese sentido, el tao es inexplicable [18, 19].

\subsection{Cambios de paradigma}

Cuando una teoría establecida topa con un fenómeno que es incapaz de explicar puede aparecer una teoría capaz de explicar este nuevo fenómeno, además de explicar todo lo anterior; la nueva teoría suele ser un superconjunto de la teoría previa.

La relatividad especial es un ejemplo de teoría que extendió la mecánica newtoniana para hacer compatibles dos pilares importantes: el principio de relatividad de GALILEO y la evidencia experimental de Michelson-Morley de que la velocidad de la luz es constante. Ambas forman parte de un mismo conjunto de teorías denominado física clásica, entre las que podemos citar la mecánica newtoniana, la teoría electromagnética y la relatividad especial y general. Para extender la mecánica newtoniana a la relatividad especial, las llamadas transformaciones de LORENTZ, así como las transformaciones de MINKOWSKI, sustituyen y amplían las anteriores transformaciones de GALILEO. La diferencia crucial es que en la mecánica newtoniana el transcurso del tiempo es absoluto y en relatividad especial deja de serlo, para dar paso a otro absoluto diferente: la velocidad de la luz. ALBERT EINSTEIN consiguió conjuntar todos estos conceptos, formulando la relatividad especial y, más tarde, incluyendo los sistemas de referencia acelerados, formulando la relatividad general.

En ocasiones, la fisura entre una teoría establecida y la teoría que la mejora es una ruptura crítica. Se necesita un cambio de conceptos tan drástico que en ese caso sucede lo que ThOMAS S. KUHN llama un cambio de paradigma o conjunto de compromisos compartidos por una comunidad científica [8]. Según KUHN, con la teoría atascada se produce una crisis, seguida de una revolución científica, que conlleva un cambio de paradigma. Como ejemplo, a finales del siglo XIX el comportamiento de los gases radiados con luz en determinadas circunstancias no tenía explicación dentro del marco de la física clásica. Este hecho, dramático en la historia de la física, vino a llamarse la catástrofe ultravioleta. La física se encontraba en una difícil encrucijada.

El nuevo paradigma que iba a poder explicar este fenómeno fue la física cuántica. Fue fraguada por numerosos científicos como MAX PLANCK, NIELS BOHR, 
ERWIN SCHRÖDINGER y WERNER HEISENBERG entre otros y a ella contribuyó de forma importante EINSTEIN, a pesar de ser un gran detractor de dicha teoría.

La física cuántica explica el mundo microscópico y desafía considerablemente el sentido común. En el mundo cuántico las partículas elementales pueden comportarse como partícula o como onda según las circunstancias. Pueden atravesar barreras, pueden tener comportamientos inexplicables en sentido clásico. En física cuántica se trabaja con ondas de probabilidad y las propiedades observables se producen por lo que se llama colapso de la función de onda [22]. Por ejemplo, el momento magnético de un un electrón no tiene asignada una orientación (arriba o abajo) hasta que no lo medimos y antes de medirlo sus propiedades están de algún modo indeterminadas, latentes. De este modo, observador y observable quedan ligados. El conocido como principio de indeterminación o incertidumbre de HEISENBERG impone una limitación natural para medir simultáneamente magnitudes como la posición y la velocidad de las partículas elementales. Todo ello, en conjunto, desafía el sentido común. Sin embargo, la física cuántica es falsable, es decir, está estructurada de forma que sus predicciones se pueden someter a pruebas experimentales que hasta la fecha han sido confirmadas con niveles de precisión experimental inigualados por otras teorías científicas.

A EINSTEIN no le gustaba este carácter indeterminista en la descripción cuántica de la naturaleza y acuñó la famosa frase «Dios no juega a los dados» para manifestar su aversión por las implicaciones del nuevo paradigma cuántico. Participó arduamente en el diseño de experimentos ideales (Gedankenexperimente, término acuñado por HANS CHRISTIAN ØRSTED y utilizado después por ERNST MACH) para demostrar inconsistencias en la teoría cuántica. Estos Gedankenexperimente, curiosamente, contribuyeron más bien al reforzamiento de la misma, lo que puede servir de buen ejemplo de puesta en práctica del ejercicio de falsacionismo. El grupo de BOHR tenía que responder a los desafíos planteados por los detractores de la teoría cuántica y para ello reconsideraban una y otra vez todos los principios de la teoría, de modo que se fue consolidando así el nuevo paradigma. El carácter indeterminista de la física cuántica depende del aspecto de la teoría que se considere: la ecuación de SCHRÖDINGER es determinista en el sentido de LAPLACE y, por otro lado, el principio de incertidumbre de HEISENBERG impone un límite fundamental al determinismo clásico.

Las teorías emergentes a partir de la física cuántica han tenido un éxito rotundo y en ellas se basa una buena parte de nuestro mundo científico y tecnológico actual: si nos damos cuenta de que los ordenadores se basan en la física cuántica no es necesario continuar justificando su importancia. Son particularmente destacables la electrodinámica [7] y la cromodinámica cuánticas (i.e. el modelo quark [3, 21]).

Tanto la física cuántica como la física del caos son popularmente conocidas a día de hoy. La física del caos tuvo su nacimiento en la misma época que la mecánica cuántica pero no tuvo un desarrollo tan temprano ni un éxito tan fulgurante como la segunda. Ambas teorías han planteado importantes limitaciones al paradigma determinista.

\section{$2.4 \quad$ Física del caos}

¿Cuándo y cómo se fraguó el paradigma del caos? A finales del siglo XIX HENRI POINCARÉ, matemático y físico francés, intentaba estudiar la estabilidad del sistema solar como sistema dinámico. Llegó a una conclusión dramática, a partir del descubrimiento de un hecho aparentemente de poca importancia: demostró que el llamado problema de los tres cuerpos no tiene solución analítica [5]. En física clásica el movimiento relativo de la Tierra y el Sol, despreciando los efectos gravitatorios de otros cuerpos celestes, es un ejemplo de problema de dos cuerpos, que tiene solución analítica como demostró NEWTON [16]. Cuando añadimos la Luna al escenario, un tercer cuerpo, el problema ya no es resoluble en los mismos términos, según demostró POINCARÉ. De hecho, en física clásica hay un gran número de problemas reales que se han de solucionar por métodos de aproximación, al no ser posible obtener soluciones analíticas para ellos. En algunos casos las aproximaciones lineales a problemas no lineales, como se explicará en la sección 5.1.6 en la página 55, pueden estar justificadas y llegar a tener un éxito rotundo suficiente, por ejemplo, para enviar cohetes a la Luna, hacerlos alunizar y traerlos de vuelta. Pero a pesar de ello la física clásica no puede dar una descripción exacta ni completa a una gran variedad de fenómenos asociados a cualquier sistema con más de dos cuerpos o problemas no lineales en general. 


\begin{tabular}{|c|c|c|c|}
\hline Paradigma & Carácter & Conceptos clave & Algunas teorías \\
\hline Física clásica & Determinista & Trayectoria & $\begin{array}{l}\text { Gravitación newtoniana, } \\
\text { relatividad }\end{array}$ \\
\hline Física cuántica & Determinista e indeterminista & Onda de probabilidad & $\begin{array}{l}\text { Electrodinámica y } \\
\text { cromodinámica cuánticas }\end{array}$ \\
\hline Física del caos & $\begin{array}{l}\text { Determinista formalmente, pero } \\
\text { limitaciones en la predecibilidad }\end{array}$ & Atractor & $\begin{array}{c}\text { Termodinámica no lineal, } \\
\text { turbulencia }\end{array}$ \\
\hline
\end{tabular}

Tabla 2.2: Paradigmas en física

Cuando hablamos de caos nos referimos a una situación aparentemente contradictoria: estudiando la evolución temporal de un sistema determinista nos encontramos con la imposibilidad de predecir su comportamiento en el largo plazo. Es como si, aceptando la existencia del demonio de LAPLACE, éste no fuera capaz de reproducir sus predicciones de futuro, proporcionando resultados diferentes cada vez que lo interrogáramos. La evolución de los sistemas caóticos no sólo no es periódica, como cabría esperar en algunos sistemas dinámicos que nos resultan familiares (sec. 5.1.5 en la página 54) sino que, además, dicha evolución muestra una sensibilidad extrema a la elección de las condiciones iniciales particulares que impongamos a los sistemas.

Desde el punto de vista físico-matemático puro el caos presenta propiedades complicadas de entender desde el punto de vista clásico. Podemos decir que el caos está asociado a fenómenos que, sin ser de naturaleza aleatoria, parecen aleatorios porque son impredecibles en la práctica. Son fenómenos en los que, si cambiamos muy ligeramente sus condiciones iniciales, su evolución futura puede cambiar drásticamente. Estos comportamientos no estaban recogidos en la mecánica newtoniana. Así pues, PoINCARÉ sembró la semilla de otro cambio de paradigma. Topó con una fisura diferente a la que produjo el paradigma cuántico y provocó un avance en una dirección distinta, que tardó en fraguarse mucho más tiempo que la teoría cuántica. Casi un siglo después se ha ido viendo la importancia del descubrimiento de POINCARÉ: dio soporte matemático formal a algo que probablemente se sospechaba desde hacía siglos y es que, de algún modo, el futuro es impredecible. O, dicho de modo menos dramático, que la posibilidad de predecir el futuro está limitada. En la Tabla 2.2 se resumen los tres paradigmas abordados aquí: el clásico, el cuántico y el del caos.

Para completar esta introducción histórica, señalamos un paralelismo entre dos hitos del desarrollo de dos ciencias como las matemáticas y la física. Así como en física el paradigma del caos supone una transformación esencial, el teorema de GöDEL (ver párrafo siguiente) supuso un punto de inflexión en la historia de las matemáticas: en uno y otro caso ambas ciencias encontraban, y afrontaban, sus propias limitaciones.

Los teoremas de incompletitud de GöDEL. En el primer tercio del siglo XX se estaba intentando renovar los fundamentos de la matemática. Dentro de ese contexto DAVID HILBERT, entre otros desafíos, planteó un programa que trataba de probar la consistencia de la teoría axiomática de conjuntos, lo que cimentaría la lógica matemática que, a su vez, soportaría el resto de ramas de las matemáticas. Sin embargo, el matemático checo KURT GöDEL estableció en 1931 que este programa no podría realizarse. Demostró, por un lado, que si la teoría axiomática de conjuntos es consistente, existen de todos modos teoremas que no pueden ser probados ni refutados, a los que llamó indecidibles (es decir, no puede haber ninguna teoría consistente y completa, particularmente cuando el proceso de deducción puede implantarse mediante un algoritmo). Por otro lado, demostró que no existe ningún procedimiento constructivo que pruebe que la teoría axiomática de conjuntos es consistente, lo que en el fondo es un caso particular del primer resultado. GÖDEL amplió la concepción del mundo matemático: las matemáticas no son un cuerpo cerrado de conocimiento donde todo es consistente, sino que hay enunciados indecidibles. La demostración del teorema es elegante y explícita, pues genera una correspondencia entre los números naturales y los símbolos de los teoremas. Esta concepción conecta, además, de forma directa con las máquinas de TURING, máquinas que teóricamente permitirían al demonio determinista de LAPLACE avanzar en su tarea de cálculo y predicción del futuro (además de distinguir entre una inteligencia artificial y una persona).

Los teoremas de incompletitud de GöDEL son uno de los resultados más profundos e impactantes de la lógica matemática porque, dentro del marco de su propio lenguaje, identificaron y pusieron de manifiesto sus propias limitaciones. 
Dentro de ese desarrollo evolutivo las ciencias van ampliando su lenguaje y sus paradigmas de modo que, cuando dentro del contexto de ese lenguaje encuentran sus limitaciones, ese lenguaje necesita ampliarse y los paradigmas sucederse. Con la teoría del caos la dinámica de sistemas encuentra, y afronta, sus propias limitaciones y con los teoremas de incompletitud de GöDEL la lógica matemática encuentra y afronta las suyas. En estos hitos se encuentra una gran belleza, una forma ejemplar del ejercicio del método científico, que encuentra sus limitaciones con su propio lenguaje y que, aceptando esas limitaciones, es capaz de crecer mediante nuevos paradigmas para seguir un camino

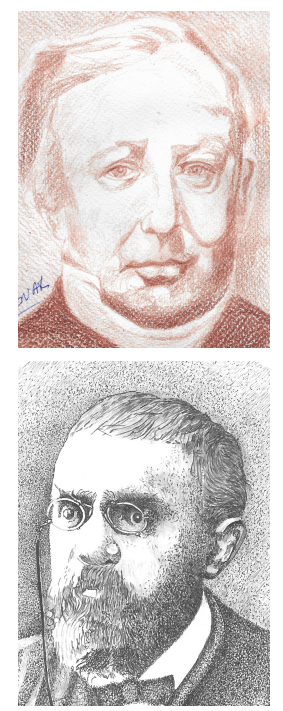

JoSEPH LiOUVILLE (1809-1882), matemático y físico francés, contribuyó en teoría de números, análisis complejo, topología diferencial, física matemática y astronomía. El teorema de Liouville de mecánica hamiltoniana tiene una estrecha relación con la teoría del caos.

HENRI PoINCARÉ (1854-1912), matemático, físico y filósofo francés, fundador de la teoría del caos, demostró que el "problema de los tres cuerpos" no tiene solución analítica. Probablemente el último ser humano capaz de abarcar todos los ámbitos de la matemática.

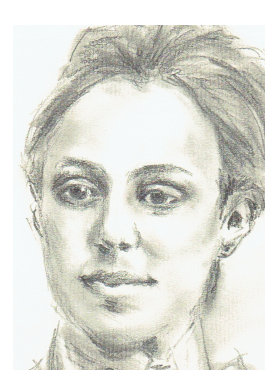

EMMY NoETHER (1882-1935),

matemática alemana, contribuyó en física teórica y álgebra abstracta, quizá la mujer más importante en la historia de la matemática. El teorema de NOETHER, que conecta simetrías y leyes de conservación, es uno de los más profundos en la historia de la física teórica. que nunca está marcado de antemano.

Desde el punto de vista de los sistemas dinámicos, en el capítulo 5 en la página 49 dedicado a la física del caos abordaremos las ideas de la teoría del caos más relevantes para la predicción meteorológica.

\subsection{Personalidades en caos}

Para finalizar este capítulo las Figuras 2.1 y 2.2 presentan una selección cronológica de personalidades vinculadas a la teoría del caos.

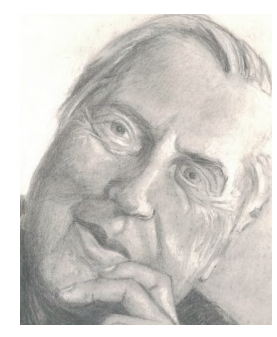

ILYA Romanovich PRIgogine (1917-2003), físico-químico belga, Premio Nobel de Química por su trabajo en estructuras disipativas, sistemas complejos e irreversibilidad.

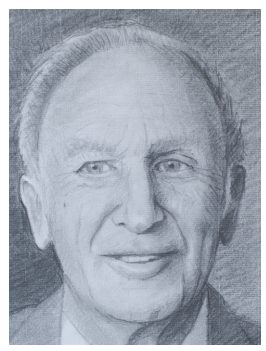

\section{EDWARD NORTON LORENZ}

(1917-2008), matemático y meteorólogo estadounidense, formuló el concepto de atractor extraño e introdujo el caos en meteorología, acuñando la idea de efecto mariposa inspirado en ideas similares de otros físicos.

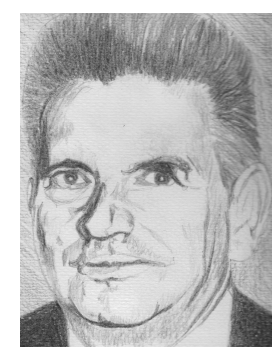

RENÉ THOM (1923-2002), matemático francés, fundador de la llamada teoría de catástrofes, muy relacionada con el caos, las singularidades y las ecuaciones diferenciales.

Figura 2.1: Personalidades en la teoría del caos 


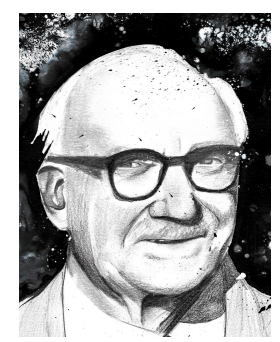

JOHN EDENSOR LITTLEWOOD

(1885-1977), matemático británico, colaborador de G. H. HARDY (tutor del matemático hindú SRINISAVA

RAMANUJAN), fue precursor de la teoría de sistemas dinámicos.

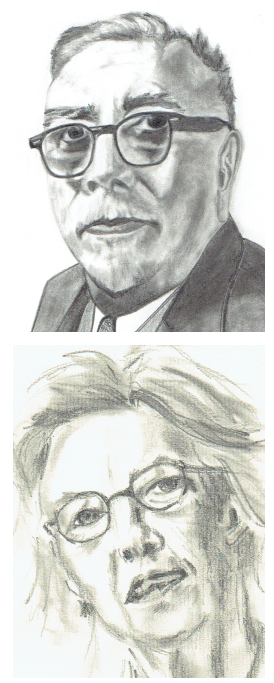

Norbert WIENER (1894-1964), matemático y filósofo estadounidense, uno de los primeros investigadores de procesos estocásticos, i.e. relacionados con el carácter aleatorio, considerado fundador de la cibernética.

\section{MARY CARTWRIGHT (1900-1998),} matemática británica y presidenta de la London Mathematical Society, fue la primera persona en analizar un sistema dinámico con teoría del caos.

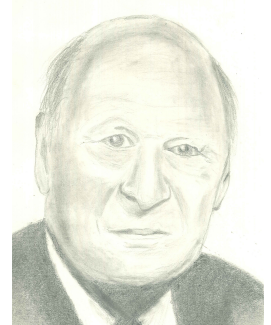

LARS ONSAGER (1903-1976),

físico-químico noruego-estadounidense, Premio Nobel de Química por las relaciones de reciprocidad en la termodinámica de procesos irreversibles.

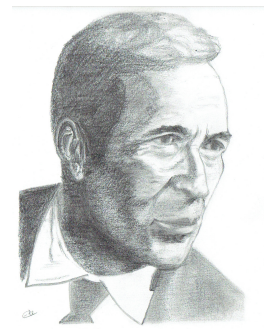

ANDREY KOLMOGOROV (1903-1987), matemático ruso, contribuyó a la teoría de probabilidades, topología, lógica, teoría de la información, complejidad computacional y, en relación con el caos, a la teoría de la turbulencia.

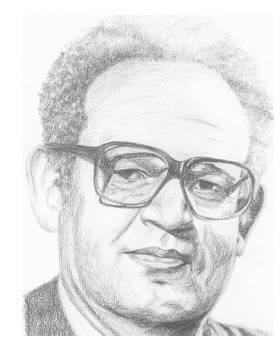

Benoit MANDElbRot (1924-2010), matemático

polaco-franco-estadounidense, investigó las estructuras autosemejantes y acuñó el término fractal.

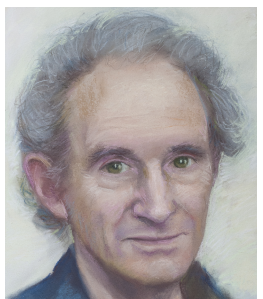

Robert MCCREdie MAY (1936-), ecólogo australiano, relacionó la teoría del caos con la dinámica de poblaciones, usando la llamada ecuación logística discreta.

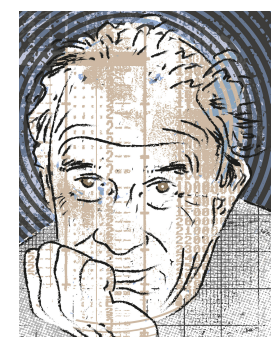

VLADIMIR IGOREVICH ARNOLD (1937-2010) matemático ruso conocido por la estabilidad de sistemas integrables, sistemas dinámicos, teoría de catástrofes, topología, ecuaciones diferenciales, hidrodinámica y singularidades.

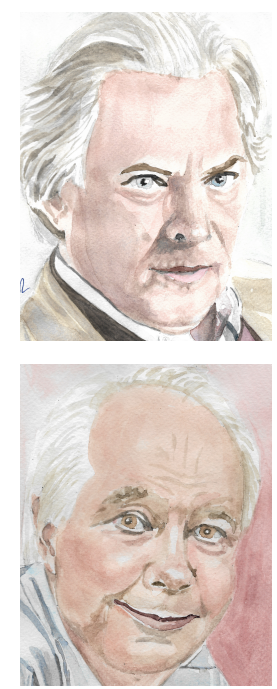

Mitchell Jay Feigenbaum (1944-) físico-matemático estadounidense, investigó en teoría de bifurcaciones y diseñó algoritmos para relacionar el caos con la cartografía y así facilitar la elaboración de mapas geográficos.

IAN STEWART (1945-), matemático británico, introdujo el llamado atractor de STEWART, contribuyó a la teoría de catástrofes y es un gran divulgador de la teoría del caos.

Figura 2.2: Personalidades en la teoría del caos. 


\subsection{Referencias}

[1] BACON, Francis. Novum organum. Clarendon press, 1878 (citado en página 14).

[2] Bunge, Mario. La ciencia, su método y su filosofía. Sudamericana, 2014 (citado en página 14).

[3] Casaus, Jorge, RodríGuez, Javier y SÁnCHEZ, Eusebio. "Cromodinámica Cuántica: el color de los quarks". En: Fotón 1 (2005), página 137 (citado en página 17).

[4] Chalmers, Alan F y col. ¿Qué es esa cosa llamada ciencia? Siglo XXI de España, 2000 (citado en página 14).

[5] Delshams, Amadeu. Poincaré, Creador de los métodos todavía modernos en las ecuaciones diferenciales y en la mecánica celeste. 2005 (citado en página 17).

[6] Feyerabend, Paul K. Tratado contra el método : esquema de una teoría anarquista del conocimiento. Tecnos, 1981. ISBN: 9788430908875 (citado en página 14).

[7] Feynman, Richard Phillips. Electrodinámica cuántica: la extraña teoría de la luz y la materia. Alianza Editorial, 1988 (citado en página 17).

[8] Kunn, Thomas Samuel. La estructura de las revoluciones científicas. Fondo de cultura económica, 2011 (citado en página 16).

[9] Lakatos, Imre., WorRall, John. y CuRRIE, Gregory. Escritos filosóficos. Alianza, 2007. ISBN: 9788420687216 . URL: https: / / dialnet . unirioja. es / servlet / libro ? codigo $=292158$ (citado en página 14).

[10] LaPlace, P. S. de. "Théorie analytique des probabilités (Introduction)". En: GauthierVillars: Paris (1817) (citado en página 16).

[11] Lloyd, Seth. "Computational Capacity of the Universe". En: Physical Review Letters 88.23 (mayo de 2002), página 237901. ISSN:
0031-9007. DOI: 10 . 1103/PhysRevLett . 88.237901 (citado en página 16).

[12] MAYO, Deborah G. Error and the growth of experimental knowledge. University of Chicago Press, 1996, página 493. ISBN: 9780226511986 (citado en página 14).

[13] Mayo, Deborah G. y Hollander, Rachelle D. Acceptable Evidence: Science and Values in Risk Management. Oxford University Press, USA, 1994, página 305. ISBN: 9780195358322 (citado en página 14).

[14] Minkel, J R. "Focus: If the Universe Were a Computer". En: Physics 9 (2002), página 27 (citado en página 16).

[15] MORIn, Edgar. "Restricted complexity, general complexity". En: Science and us: Philosophy and Complexity. Singapore: World Scientific (2007), páginas 1-25 (citado en página 14).

[16] Newton, Isaac y col. Philosophiae naturalis principia mathematica. Volumen 1. excudit G. Brookman; impensis TT et J. Tegg, Londini, 1833 (citado en página 17).

[17] PopPer, Karl R. Conjeturas y refutaciones: el desarrollo del conocimiento científico. Paidós Ibérica, 1991 (citado en página 14).

[18] SuZUKI, Daisetz Teitaro. El zen y la cultura japonesa. RBA, 2002. ISBN: 8447325148. URL: https: //dialnet .unirioja.es / servlet / libro? codigo=93161 (citado en página 16$)$.

[19] TsE, Lao. Tao te ching. Mauad Editora Ltda, 2011 (citado en página 16).

[20] Ulanowicz, Robert E. Growth and development: ecosystems phenomenology. Springer Science \& Business Media, 2012 (citado en página 16$)$.

[21] Weinberg, Steven y Ron, José Manuel Sánchez. Los tres primeros minutos del universo. Alianza Editorial, 1996 (citado en página 17). 
[22] Wilber, Ken. Cuestiones cuánticas: W. Heisenberg, E. Schrödinger, A. Einstein, Sir J. Jeans, M. Planck, W. Pauli, sir A. Edding- ton: escritos místicos de los físicos más famosos del mundo. Kairós, 1994 (citado en página 17). 